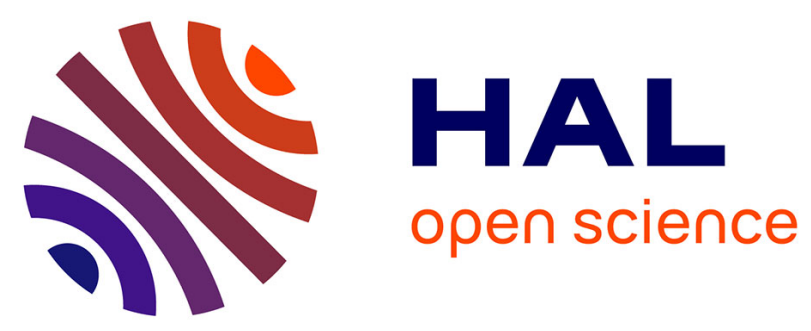

\title{
Dependence of properties of magnetospheric line radiation and quasiperiodic emissions on solar wind parameters and geomagnetic activity
}

B. Bezděková, F. Němecc, Michel Parrot, M. Hajoš, J. Záhlava, O. Santolik

\section{- To cite this version:}

B. Bezděková, F. Němecc, Michel Parrot, M. Hajoš, J. Záhlava, et al.. Dependence of properties of magnetospheric line radiation and quasiperiodic emissions on solar wind parameters and geomagnetic activity. Journal of Geophysical Research Space Physics, 2019, 124 (4), pp.2552-2568. 10.1029/2018JA026378 . insu-02086020

\section{HAL Id: insu-02086020 \\ https://hal-insu.archives-ouvertes.fr/insu-02086020}

Submitted on 6 Sep 2019

HAL is a multi-disciplinary open access archive for the deposit and dissemination of scientific research documents, whether they are published or not. The documents may come from teaching and research institutions in France or abroad, or from public or private research centers.
L'archive ouverte pluridisciplinaire HAL, est destinée au dépôt et à la diffusion de documents scientifiques de niveau recherche, publiés ou non, émanant des établissements d'enseignement et de recherche français ou étrangers, des laboratoires publics ou privés. 


\section{JGR Space Physics}

\author{
RESEARCH ARTICLE \\ 10.1029/2018JA026378 \\ Key Points: \\ - Dependences obtained for \\ quasiperiodic emissions with \\ short/long modulation periods are \\ different \\ - Properties of quasiperiodic emissions \\ and magnetospheric line radiation \\ observed at the same times are not \\ related \\ - Event properties are not affected by \\ the whistler occurrence
}

Correspondence to:

B. Bezděková,

baja@etranslator.biz

Citation:

Bezděková, B., Němec, F., Parrot, M., Hajoš, M., Záhlava, J., \& Santolík, O. (2019). Dependence of properties of magnetospheric line radiation and quasiperiodic emissions on solar wind parameters and geomagnetic activity. Journal of Geophysical Research: Space Physics, 124, 2552-2568. https://doi.org/10.1029/ 2018JA026378

Received 6 DEC 2018 Accepted 18 MAR 2019 Accepted article online 27 MAR 2019 Published online 6 APR 2019
(C)2019. American Geophysical Union. All Rights Reserved.

\section{Dependence of Properties of Magnetospheric Line Radiation and Quasiperiodic Emissions on Solar Wind Parameters and Geomagnetic Activity}

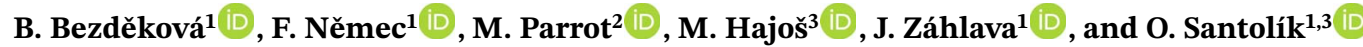 \\ ${ }^{1}$ Faculty of Mathematics and Physics, Charles University, Prague, Czech Republic, ${ }^{2}$ LPC2E/CNRS, Orléans, France, \\ ${ }^{3}$ Department of Space Physics, Institute of Atmospheric Physics, The Czech Academy of Sciences, Prague, \\ Czech Republic
}

\begin{abstract}
Very low frequency electromagnetic waves in the inner magnetosphere sometimes exhibit either frequency or time modulation. These phenomena are called, respectively, magnetospheric line radiation (MLR) and quasiperiodic (QP) emissions. Data from Detection of Electro-Magnetic Emissions Transmitted from Earthquake Regions spacecraft were used to analyze their properties, such as MLR frequency spacing, QP modulation period, and QP intensity as functions of geomagnetic activity and solar wind parameters. Altogether, 1,152 MLR events and 2,172 QP emissions were analyzed. It is shown that the influence of the analyzed parameters on QP emissions is different for QP events with modulation periods shorter/longer than $20 \mathrm{~s}$. While the properties of QP events with long modulation periods are significantly related to the geomagnetic activity and solar wind parameters, no such dependences are observed for events with short modulation periods. This suggests that there might be two types of QP emissions generated by two different mechanisms. It is further shown that there seems to be no relation between the properties of QP and MLR events observed at the same times. Finally, the event properties do not seem to be related to the whistler occurrence rate.
\end{abstract}

\section{Introduction}

Whistler mode electromagnetic waves observed in the very low frequency range in the inner magnetosphere are sometimes modulated either in the frequency or time domain. Such waves are called magnetospheric line radiation (MLR) and quasiperiodic (QP) emissions, respectively.

MLR events are usually observed at frequencies from about 1 to about $8 \mathrm{kHz}$ (Helliwell et al., 1975). Their frequency-time spectrograms are formed by several almost parallel and nearly horizontal intense lines. Although such events have been observed both on the ground (e.g., Helliwell et al., 1975; Rodger et al., 1999, 2000b) and in space (e.g., Bell et al., 1982; Němec et al., 2012; Parrot et al., 2005; Rodger et al., 1995), their origin is not yet clarified. Bullough (1995) discussed a possible relation of MLR events to power line harmonic radiation. However, the frequency spacings of the events observed at Halley, Antarctica, do not correspond to harmonics of electric power system (Rodger et al., 1999) and the events are thus most likely of a natural origin (Rodger et al., 2000a). Ground-based observations revealed typical event durations of about $30 \mathrm{~min}$ and their predominant occurrence after periods of enhanced geomagnetic activity (Rodger et al., 2000b).

The first systematic analysis of MLR events observed by spacecraft was published by Rodger et al. (1995). They used International Satellites for Ionospheric Studies 1 and 2 to demonstrate the existence of two different types of MLR events. The first type of events is formed by broadband spectral lines with an observable frequency drift (on the order of a few tens of hertz per second) with frequency spacings not corresponding to the multiples of 50 or $60 \mathrm{~Hz}$. In view of later studies (e.g., Němec et al., 2007), only this type of events will be considered as MLR events in this study. The emissions of the second type are formed by spectral lines with narrow bandwidths with principally no frequency drift and frequency spacings close to the multiples of either 50 or $60 \mathrm{~Hz}$. This type of events is nowadays most often classified as power line harmonic radiation (e.g., Němec et al., 2007).

Surveys of MLR events observed by the low-altitude Detection of Electro-Magnetic Emissions Transmitted from Earthquake Regions (DEMETER) spacecraft were presented by Němec et al. (2009) and Němec et al. 
(2012). They showed that the events were observed more frequently during the day than during the night and predominantly during or after the periods of enhanced geomagnetic activity. The frequency spacing was found to be larger at the times of lower radial distances of model plasmapause. The frequency drift of the events was shown to be usually positive and neither the frequency drift nor the frequency spacing were related to the L-shell where the events were observed. The analysis of MLR events observed during the entire DEMETER mission including 1,230 MLR events was reported by Bezděková et al. (2015). This study shows that the events are observed more likely between November and April, their occurrence is statistically related to specific variations of solar wind parameters, and it is significantly lower at geomagnetic longitudes corresponding to the South Atlantic Anomaly, which indicates the importance of drift loss cone electrons for the wave occurrence. Ground-based (Manninen, 2005) and multipoint (Němec et al., 2012; Parrot et al., 2007) MLR observations reveal that the events can be quite long-lasting and observable over a spatially significantly extended region.

While MLR events are characteristic by their frequency modulation of the intensity, in the case of QP emissions the time modulation of the wave intensity is observed. They usually occur in the frequency range between about 0.5 and $4 \mathrm{kHz}$ and the time separations between consecutive QP elements (modulation periods) can vary from some tens of seconds up to a few minutes (Carson et al., 1965; Manninen et al., 2012; Sato \& Fukunishi, 1981). QP emissions are usually observed on the dayside (Engebretson et al., 2004; Hayosh et al., 2014; Martinez-Calderon et al., 2015).

QP emissions observed on ground-based stations were classified into two types according to whether or not they were accompanied by ground-based measurements of ultralow frequency (ULF) magnetic field pulsations with periods corresponding to the periods of QP emissions (Kitamura et al., 1969; Sato et al., 1974). If such ULF pulsations are present, the QP events are categorized as QP emissions type 1 and they are classified as QP emissions type 2 otherwise.

Two different mechanisms for QP events generation were proposed. The first possibility related to the QP emissions type 1 is that the source region is periodically modulated by a compressional ULF wave, which results in a QP modulation of the resonant conditions and wave growth (Chen, 1974; Sato \& Fukunishi, 1981). Moreover, Kimura (1974) showed that even weak ULF magnetic field pulsations can cause significant modulations of the source region. Nevertheless, magnetic field pulsations observed on the ground are usually Alfvénic (noncompressional), whereas this mechanism presumes the presence of a compressional ULF wave at least in the equatorial region, where the emissions are likely generated (Morrison, 1990; Němec et al., 2018)

The second possible generation mechanism of QP emissions is based on a self-consistent model of periodic wave generation in the regime of relaxation oscillations developed by Trakhtengerts et al. (1986), Demekhov and Trakhtengerts (1994), and Pasmanik et al. (2004). This model was successfully employed to explain some of the satellite observations of QP emissions (Pasmanik et al., 2004). Due to the absence of any modulating ULF wave in this model, this mechanism is suitable for describing the origin of QP emissions type 2. Note, however, that this classification of QP emissions was established according to their ground-based measurements. When spacecraft data are used instead, the distinction between individual QP types becomes significantly less clear (Tixier \& Cornilleau-Wehrlin, 1986). Sazhin and Hayakawa (1994) even suggested that both types of QP emissions could be generated by the same mechanism and their classification into the two aforementioned types is thus questionable. In any case, the suggested mechanisms were still not satisfactorily verified by observations and their origin thus remains mostly unexplained (Manninen et al., 2018).

The emissions most likely propagate unducted at least for a part of their propagation path (e.g., Gołkowski \& Inan, 2008; Hayosh et al., 2016; Němec et al., 2013). Available conjugate observations of QP events demonstrate that their modulation is the same over a large spatial extent (e.g., Martinez-Calderon et al., 2016; Němec et al., 2013, 2016; Titova et al., 2015).

Properties of QP emissions observed by the DEMETER spacecraft were analyzed by Hayosh et al. (2014). They showed that QP events with longer modulation periods tend to have lower frequency drifts and wave amplitudes. The event properties are not related to the satellite location and the event occurrence rate is lower at geomagnetic longitudes of South Atlantic Anomaly. They also demonstrated that QP modulation periods can vary by about $20 \%$ during a single event. We note, however, that the DEMETER observations 
are limited to rather short time intervals when this nearly polar orbiting spacecraft crosses the regions filled with the waves. During specific long-lasting events the ratio between the longest and the shortest observed modulation periods can be as large as about 4 (Manninen et al., 2014; Titova et al., 2015).

The properties of QP emissions can be possibly affected by lightning generated whistlers. Specifically, Ho (1973) reported an increase of the QP modulation period and a modification of the emission structure at the times of simultaneously observed lightning generated whistlers. Additionally, "'Manninen et al. (2014) observed a periodic fine structure inside individual QP elements, with periods comparable to the periods of two-hop whistler mode waves.

In this study an analysis of MLR and QP events based on the full DEMETER spacecraft data set is presented. In particular, it is investigated how their detailed properties (MLR frequency spacing, QP modulation period, QP maximal intensity) depend on the geomagnetic activity and solar wind parameters and, moreover, how they relate to each other. Section 2 presents the procedure used to obtain the MLR and QP event properties and the resulting data set. The obtained results are described in section 3, and they are discussed in section 4. Finally, the main results are summarized in section 5.

\section{Data Set}

We use the data measured by a low-altitude satellite DEMETER. This satellite operated between 2004 and 2010 on an almost Sun-synchronous circular orbit with an altitude of approximately $700 \mathrm{~km}$. Due to the Sun-synchronous orbit, the satellite measurements were always performed at a local time close to either 10:30 or 22:30 hr. Wave measurements were continuously performed at geomagnetic latitudes lower than $65^{\circ}$. The spacecraft made 14 orbits per day.

In the very low frequency range one electric field component and one magnetic field component were measured by the DEMETER spacecraft. Frequency-time spectrograms of power spectral density of these components up to $20 \mathrm{kHz}$ were calculated on board with the frequency resolution of about $19.53 \mathrm{~Hz}$ and the time resolution of about $2 \mathrm{~s}$. Because of substantial interferences in the magnetic field measurements only electric field measurements are used in this paper (Berthelier et al., 2006).

Detection and classification of lightning-generated whistlers observed by DEMETER was possible due to an on board implemented neural network (Elie et al., 1999). This allowed us to determine an average whistler occurrence rates at the times of individual events.

OMNI solar wind data propagated to the bow shock location were used to characterize solar wind parameters at the times of event observations. In this study data concerning the interplanetary magnetic field (IMF) magnitude, proton density, flow speed, and flow pressure with the time resolution of one hour were used.

\subsection{MLR Events}

Altogether, 1,230 MLR events were identified in the DEMETER data set (Bezděková et al., 2015). These events were detected in 1054 half orbits (out of 57,574 half orbits in total). Out of the events, 781 events were identified during daytime half orbits, and 449 events were identified during nighttime half orbits.

Figure 1a shows an example of the MLR event observed on 5 February 2009. The event was observed between 7:15 and 7:20 UT in the frequency range of about 3.2-4.6 kHz. The event occurred at geomagnetic latitudes of about $-54^{\circ}(L \approx 3.2)$. Altogether, 18 lines were marked for the event. Their frequency spacing is approximately $80 \mathrm{~Hz}$, and their frequency drift is approximately $0.14 \mathrm{~Hz} / \mathrm{s}$. The lines depicted in Figure $1 \mathrm{~b}$ were obtained by a manual procedure. Due to the low event intensity at the edges of the frequency range, it was sometimes difficult to mark a line properly. In such a case, it was omitted from the further analysis. These lines are dashed in Figure 1b.

The properties of MLR events analyzed in this study are their frequency drifts and frequency spacings as defined by Němec et al. (2012). In order to determine their values, individual intense lines in the frequency-time spectrograms forming the events were visually identified and fitted by straight lines. This was performed manually by a mouse pointer on a screen. An example of this "line identification" is shown in Figure 1b, which is actually a zoom of the event presented in Figure 1a. As it was sometimes not obvious whether a line segment is present or not, it was not possible to perform this type of analysis for all events. Altogether, 1,152 out of 1,230 observed events were analyzed. 

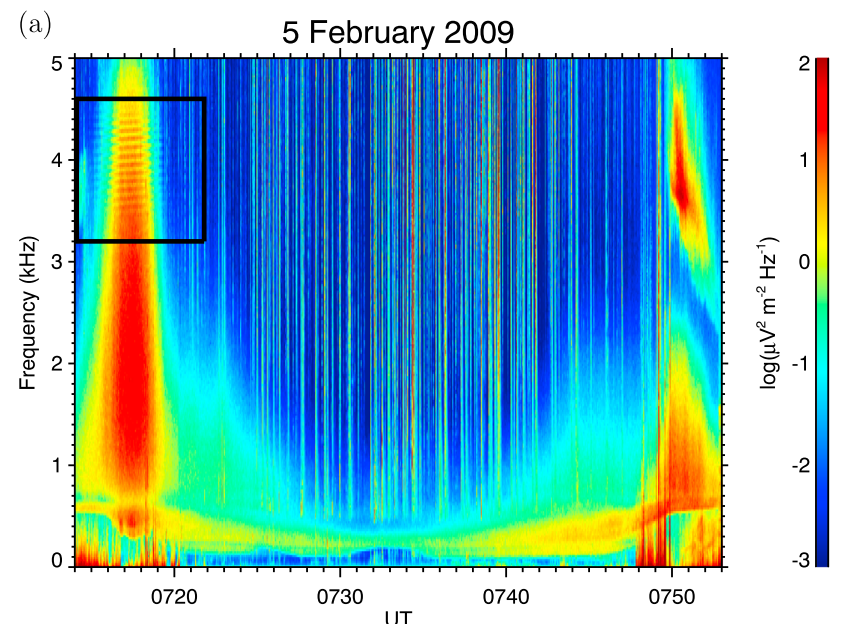

(b)

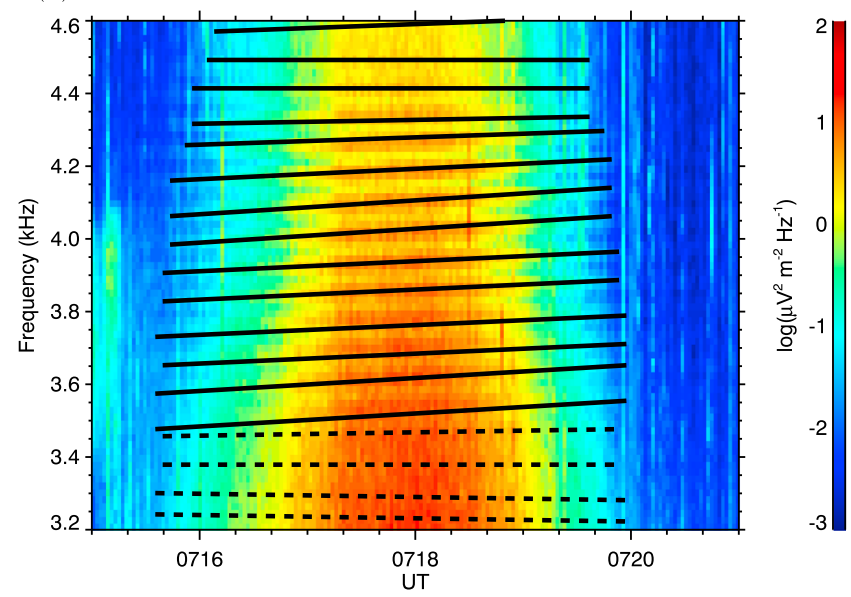

Figure 1. (a) An example of frequency-time spectrogram measured by the Detection of Electro-Magnetic Emissions Transmitted from Earthquake Regions spacecraft where an MLR event was detected. The event occurred on 5 February 2009 between about 07:15:30 and 07:20:00 UT in the frequency range between about 3.2 and $4.6 \mathrm{kHz}$ (marked by the black rectangle). (b) A zoom of the frequency-time spectrogram where the MLR event was detected. The individual lines forming the event are marked by the black lines. These were used to determine the frequency drift and the frequency spacing of the event. The dashed lines were omitted from the further analysis (see text). MLR = magnetospheric line radiation.

The identified lines forming individual MLR events were used to calculate their frequency drift and frequency spacing. Following the procedure described in detail by Němec et al. (2012), the frequency drift of an event was defined as an arithmetic average of frequency drifts of individual lines forming the event. This was used as a baseline, which was subtracted from the identified MLR lines. The frequency spacing of an event was then determined by a linear regression of the residual frequencies of individual lines forming the event. Note that both the frequency drift and the frequency spacing could slightly vary during the event duration. In such a case, our procedure effectively returns average characteristics of the event. It should be noted that the performed processing intrinsically assumes that the frequency drift of all lines forming a given event is approximately the same and that their frequency spacings do not vary too much as a function of frequency. These assumptions are typically well valid throughout our data set.

\subsection{QP Events}

The total number of QP emission events found in the DEMETER data was 2,264 (Hayosh et al., 2016). Out of them, 2,181 events were observed in the daytime half orbits and just 83 events were detected in the nighttime half orbits. Given that the events observed at low latitudes should be classified as QP modulated whistler mode equatorial noise (also known as fast magnetosonic waves) rather than QP emissions (Němec et al., 2015, 2018; Parrot et al., 2016), we consider only QP events which at least partially spanned to geomagnetic 

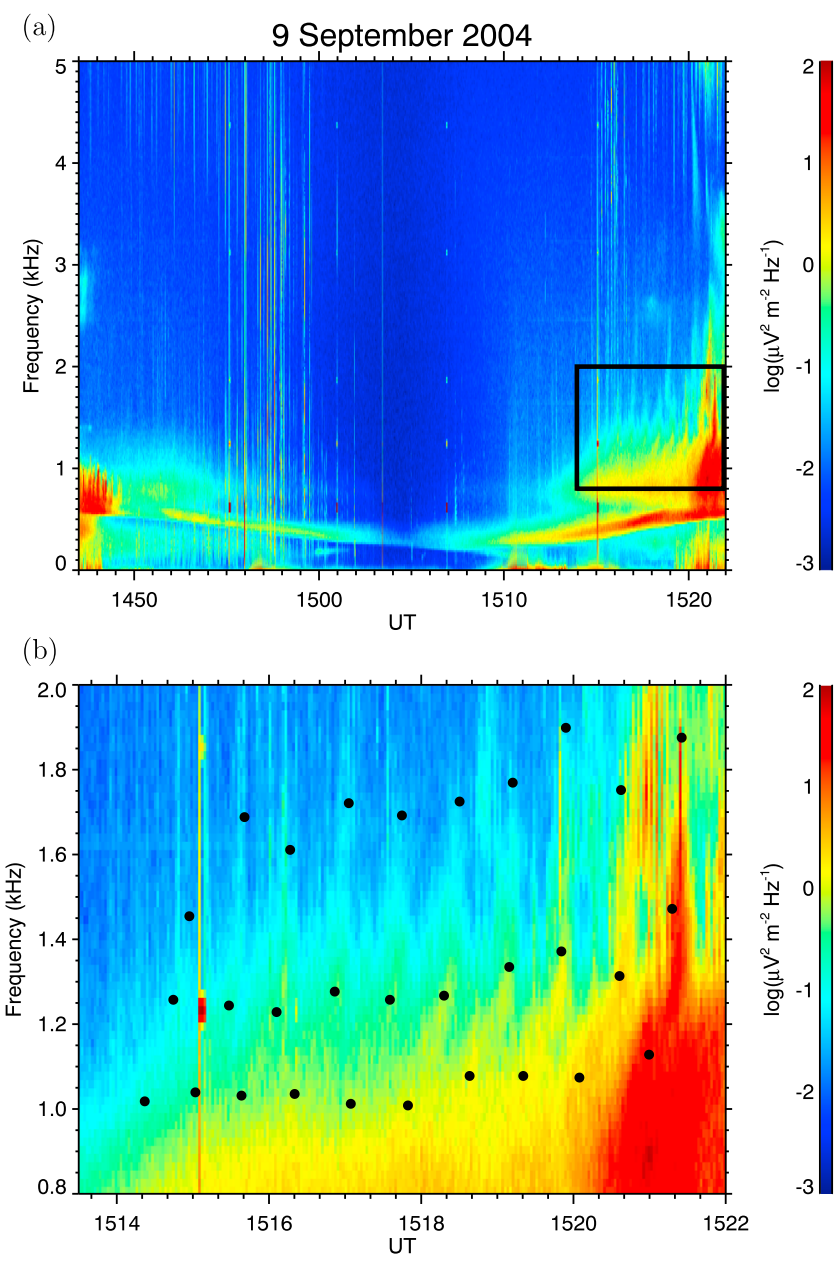

Figure 2. (a) An example of frequency-time spectrogram measured by the DEMETER spacecraft where a QP emission was detected. The event was observed on 9 September 2004 between about 15:14:20 and 15:21:30 UT in the frequency range between about 1 and $2 \mathrm{kHz}$ (marked by the black rectangle). Note that there is an additional QP event observable around 14:48 UT at frequencies of about $0.8 \mathrm{kHz}$, but it will not be discussed further. (b) A zoomed frequency-time spectrogram where QP emission was observed. The bottom and upper black points indicate beginning and ending of the individual QP elements. The middle points are the points where the frequency sweep rates of the elements change. These points were used to characterize the element slopes in order to determine their intensities and modulation periods (see text). QP = quasiperiodic.

latitudes larger than $40^{\circ}$. This leaves us with 2,172 events, out of which 79 were observed on the nightside. An example of a QP event detected by the DEMETER spacecraft on 9 September 2004 is shown in Figure 2. The event was observed between 15:14 and 15:21 UT at frequencies from about 1 to about $2 \mathrm{kHz}$ at geomagnetic latitudes of about $-50^{\circ}(L \approx 2.3)$. The period of this event is about $43 \mathrm{~s}$, and the number of detected elements is 10. An additional weak QP emission occurred between approximately 14:47 and 14:52 UT in the frequency range about $0.6-1.1 \mathrm{kHz}$, but it will not be discussed further.

The analyzed properties of QP emissions are their modulation periods and intensities following Hayosh et al. (2014). An example of a zoomed frequency-time spectrogram is shown in Figure 2b. Each QP element forming a given event was manually characterized by three points, which allows us to describe a possible change in the element sweep rate. These points were selected in such a way that the intensity at the corresponding frequency is maximal at the times of those points. The first point and the third point are placed at the lowest and the highest frequencies of the QP elements. The second (middle) point is defined by the time where the frequency sweep rate of an element changes. These three points were then connected by straight lines and frequency-time intervals intersected by those lines were used to evaluate the element intensity as a median of respective measured intensities. We further defined a "maximal intensity of QP emission" as the 

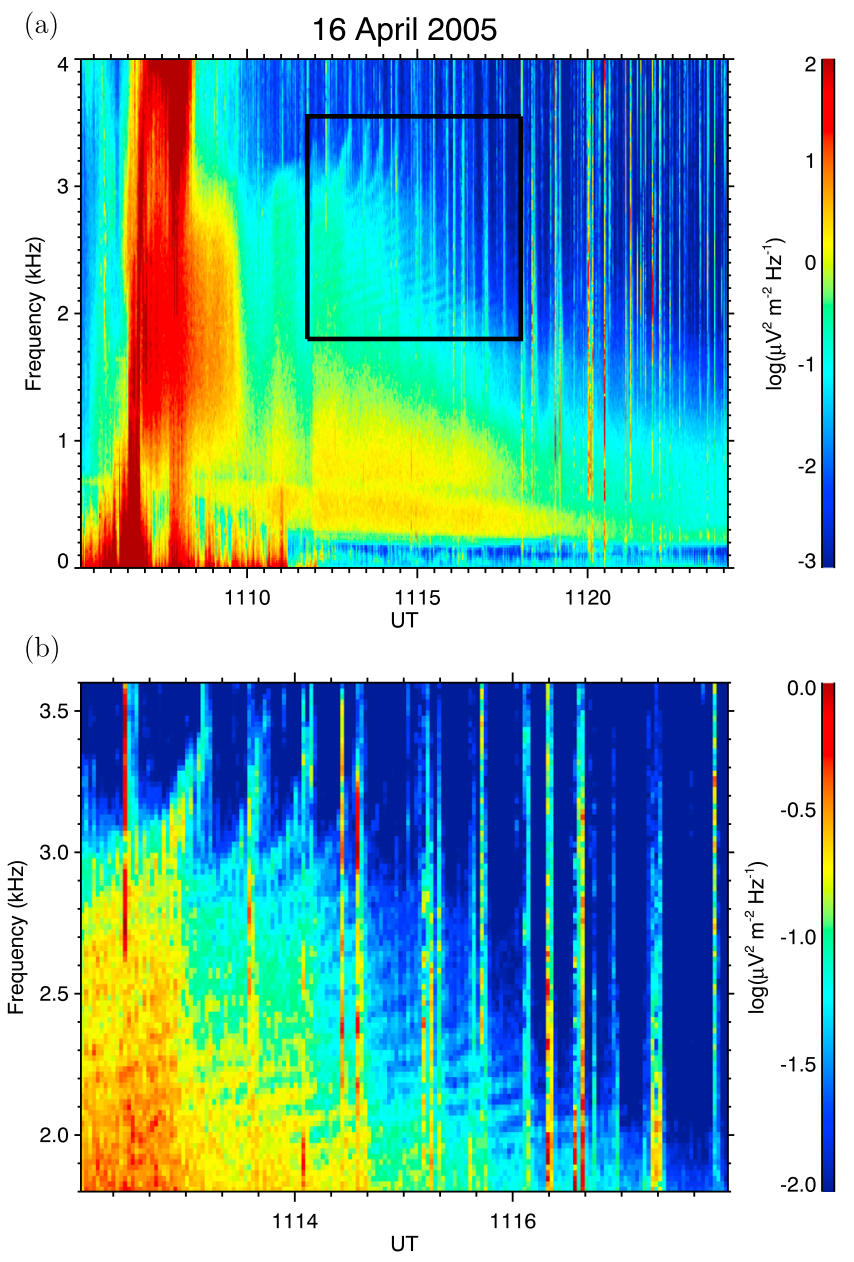

Figure 3. (a) Frequency-time spectrogram of an event whose classification as MLR/QP is rather ambiguous. The event was observed on 16 April 2005 between about 11:13 and 11:17 UT at frequencies between about 1.8 and $3.5 \mathrm{kHz}$ (marked by the black rectangle). While the observed frequency drift is rather low at low frequencies and the event might be classified as MLR, it progressively increases with the frequency and the higher frequency part of the event is more QP like. (b) A zoom of the part of the frequency-time spectrogram where the event was detected. Note that the color scale in the plot was changed in order to better highlight the elements of the event. MLR = magnetospheric line radiation; $\mathrm{QP}=$ quasiperiodic.

intensity of its most intense QP element. This was done in order to ensure that the intensity of an otherwise intense QP event would not be decreased by possible low-intensity QP elements detected on its edges. The modulation period of a QP event was defined as a median time separation between consecutive QP elements.

\subsection{Distinction Between MLR and QP}

As described above, MLR events are emissions with a nearly periodic frequency modulation of the wave intensity, while QP events are emissions with a clear temporal QP modulation of the wave intensity in the emission band. Moreover, the QP elements start from more or less the same frequency, even if the emission can be present simultaneously at lower and higher frequencies when the closest neighbor elements partially overlap, as it is the case in Figure 2.

We note that although this classification is typically quite clear, there are few outstanding cases when the distinction between the two types of events is rather ambiguous. An example of such an event is shown in Figure 3. It shows a frequency-time spectrogram obtained by DEMETER on 16 April 2005 between about 11:05 and 11:24 UT. The event marked by the black rectangle occurred between about 11:13 and 11:17 UT at frequencies between about 1.8 and $3.5 \mathrm{kHz}$. It can be seen that the frequency drifts of the intense lines forming the event are rather low at low frequencies, but they progressively increase toward higher frequencies. 


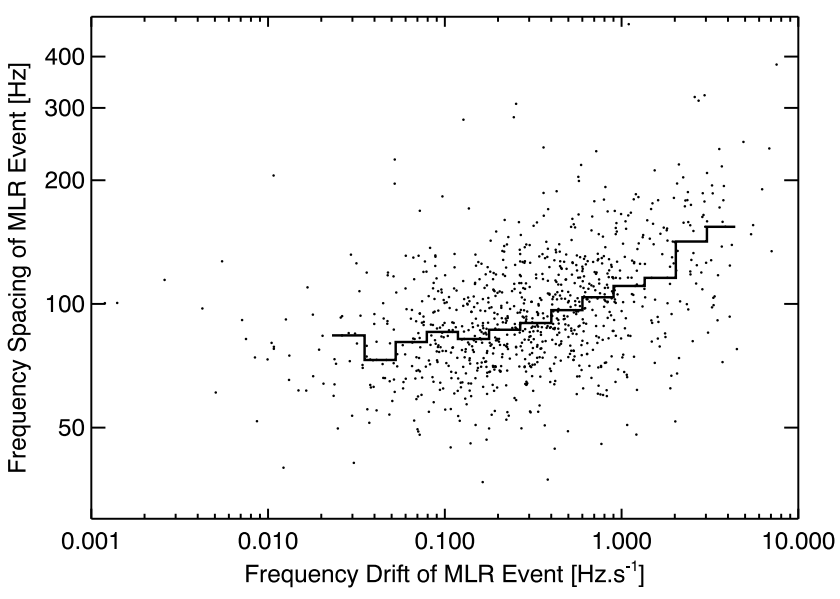

Figure 4. Dependence of MLR frequency spacing on MLR frequency drift. Only events with positive frequency drift were included $(1,000$ cases out of 1,152). Black horizontal lines correspond to median values. MLR = magnetospheric line radiation.

Consequently, while the lower frequency part of the event would be classified as MLR, the higher frequency part is more QP like.

\section{Results}

\subsection{MLR Events}

Figure 4 shows the dependence of MLR frequency spacing on MLR frequency drift. Note that occasional events with negative frequency drifts $(152$ events out of 1,152$)$ are not plotted. The median frequency spacing for these events is about $80 \mathrm{~Hz}$. It can be seen that the frequency spacing of MLR events is larger for events with larger-frequency drifts. Given the limited frequency resolution of data $(19.53 \mathrm{~Hz})$ and a typical duration of MLR events observed by DEMETER (few minutes), the evaluation of MLR frequency drift is rather inaccurate and the frequency drifts are thus omitted from most of the following analysis.

Having determined the frequency spacings for all identified MLR events, it is of interest to investigate how these depend on relevant controlling parameters. The dependences on the geomagnetic activity expressed by $\mathrm{AE}$ and Dst indices are investigated in Figure 5. Each point corresponds to a single MLR event. The horizontal black lines show the median values over corresponding multiplicative factor of 2 in AE values and over intervals of $10 \mathrm{nT}$ in Dst values. It can be seen that the median frequency spacings systematically increase with increasing geomagnetic activity (larger AE index or lower Dst index). While the median frequency spacing is as low as about $75 \mathrm{~Hz}$ at the times of low geomagnetic activity, it increases to about $120 \mathrm{~Hz}$ during severely disturbed periods. The respective rank correlation coefficients obtained for AE and Dst indices are

(a)

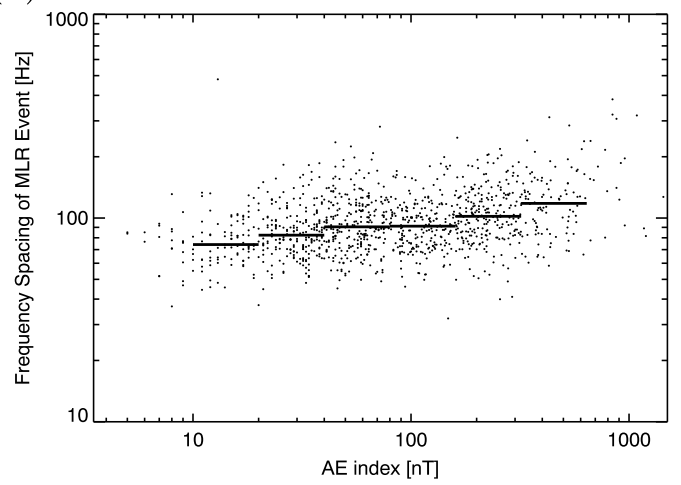

(b)

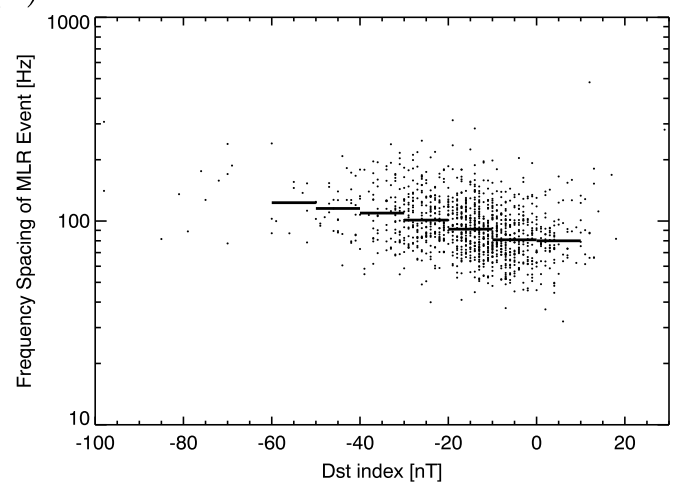

Figure 5. MLR frequency spacing as a function of the (a) AE and (b) Dst indices. Each point corresponds to a single MLR event. Horizontal black lines correspond to median values in given AE and Dst intervals. MLR = magnetospheric line radiation. 
(a)

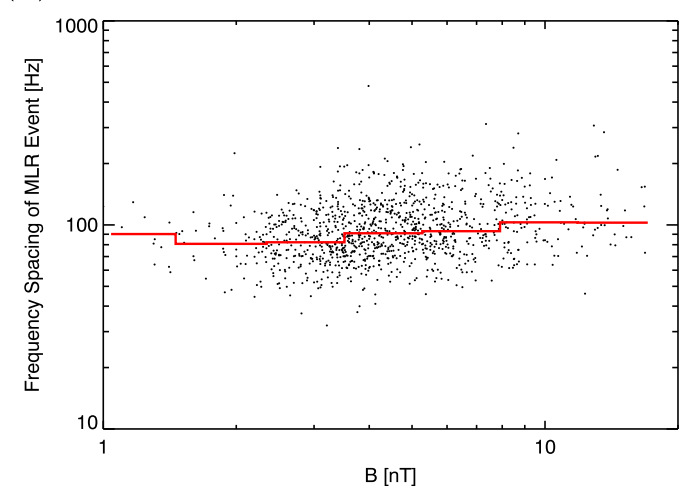

(c)

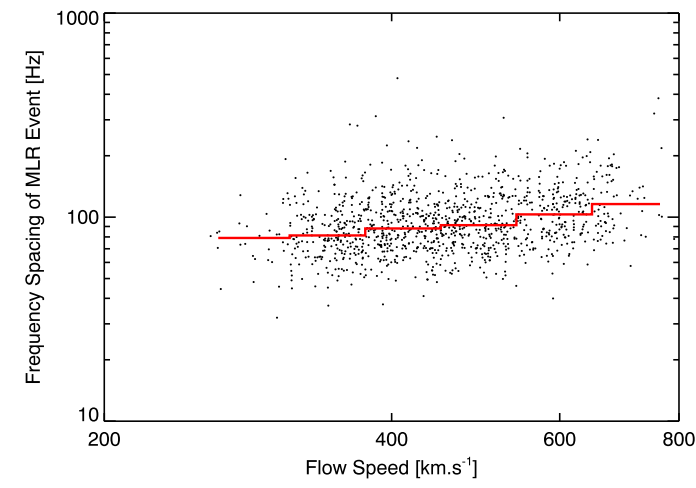

(b)

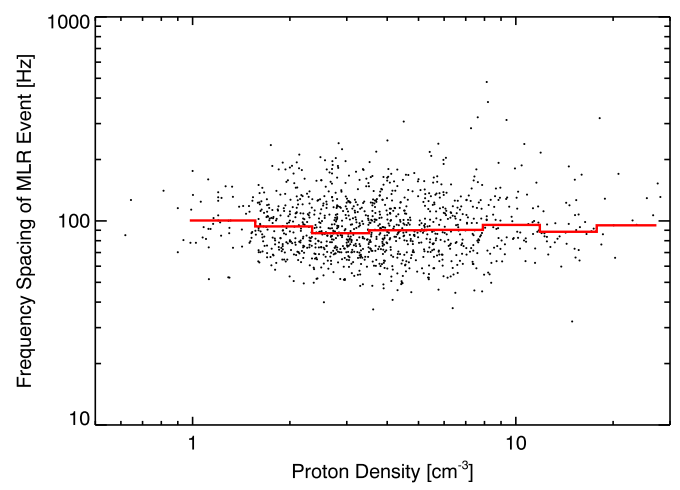

(d)

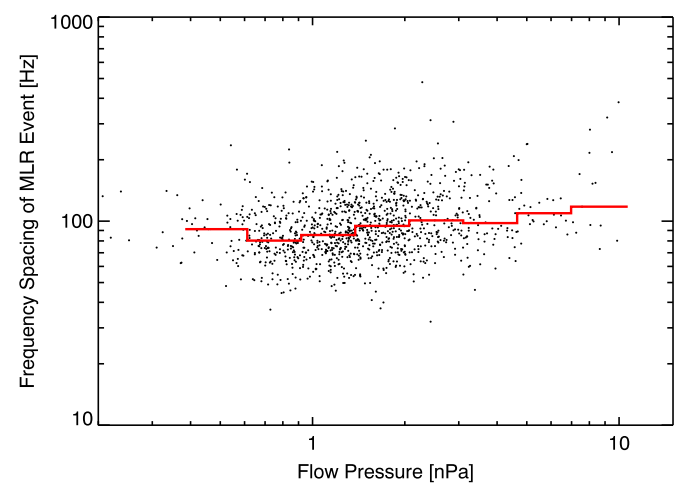

Figure 6. Dependence of the frequency spacing of MLR events on (a) interplanetary magnetic field magnitude, (b) solar wind proton density, (c) solar wind flow speed, and (d) solar wind flow pressure. Black points correspond to values obtained for individual MLR events. Red horizontal lines mark the median values of MLR frequency spacings in given intervals of solar wind parameters. MLR = magnetospheric line radiation.

about 0.34 and -0.35 , respectively. However, the random scatter of individual data points is larger than this systematic trend and a much wider range of individual frequency spacings (from 32.16 to $479.64 \mathrm{~Hz}$ ) can be obtained during both active and calm geomagnetic conditions.

Similar analysis as for the frequency spacings of MLR events was performed also for their frequency drifts (not shown). Given that the MLR frequency spacings and frequency drifts are strongly related (see Figure 4), the obtained results correspond to the dependences obtained for the frequency spacings. However, as the determination of MLR frequency drifts is rather inaccurate, the data points exhibit a significant scatter and the appropriate rank correlations are quite low (about 0.15 and -0.18 for AE and Dst indices, respectively).

The variation of MLR frequency spacing is investigated as a function of the solar wind properties in Figure 6. Figures 6a-6d show a dependence of the MLR frequency spacing on the IMF magnitude, proton number density, flow speed, and flow dynamic pressure, respectively. The red horizontal lines correspond to the median values calculated over respective logarithmically spaced intervals of solar wind parameters (corresponding to multiplicative factors of 1.3, 1.5, 1.2, and 1.5 for IMF magnitude, proton number density, flow speed, and flow dynamic pressure, respectively). It can be seen that the MLR frequency spacing is not significantly correlated with any of the investigated solar wind parameters. There is, however, a weak tendency for the MLR frequency spacings to increase with the solar wind flow speed (rank correlation coefficient of about 0.28).

\subsection{QP Events}

A possible controlling role of the geomagnetic activity regarding the modulation period of QP emissions is investigated in Figures 7a and 7b. Each plotted point corresponds to a single QP event. Although their scatter is very significant and masking any systematic trend for each individual case, one can identify a tendency for the modulation periods to decrease with increasing geomagnetic activity in the upper part of the 
(a)

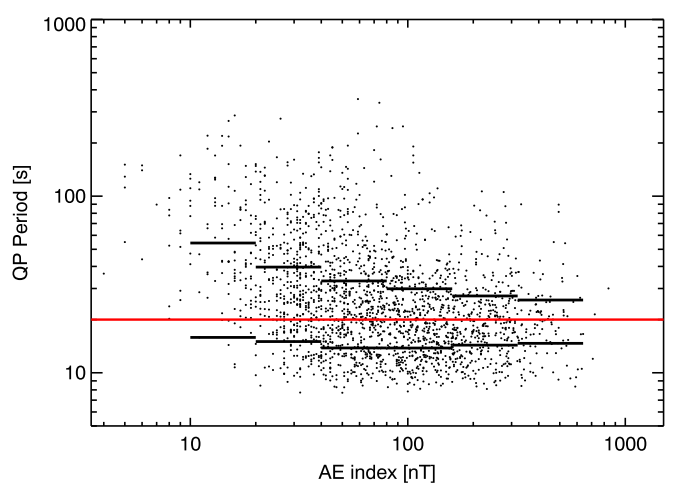

(c)

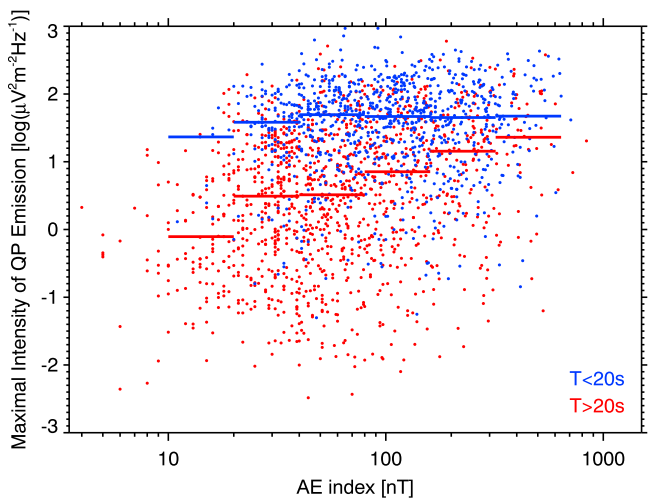

(b)

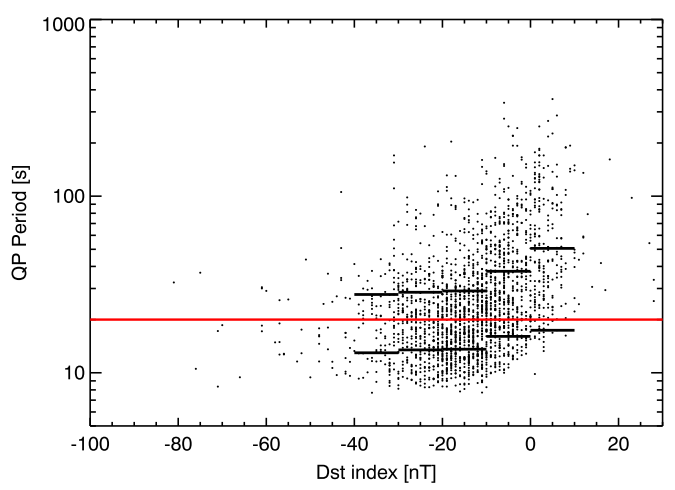

(d)

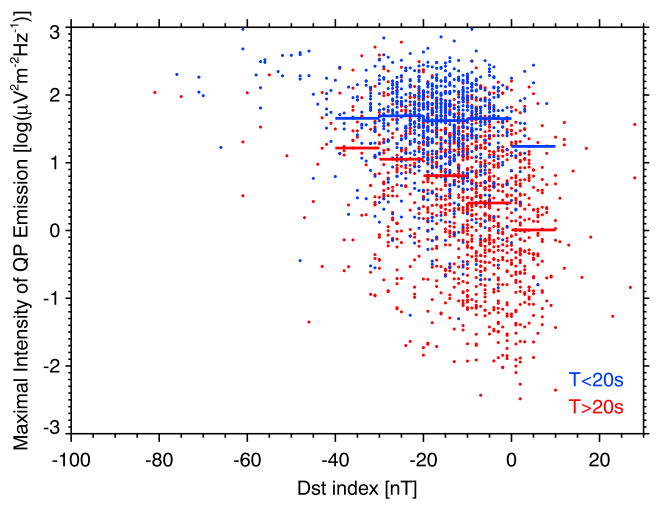

Figure 7. (a, b) QP modulation period as a function of the AE and Dst indices, respectively. Each point corresponds to a single event. The red horizontal lines mark the modulation period of $20 \mathrm{~s}$. The horizontal black lines show median modulation period values calculated separately for short and long modulation period events. (c, d) Maximal intensity of QP events as a function of the AE and Dst indices, respectively. The red points and the red median values were obtained for events with modulation periods longer than $20 \mathrm{~s}$. The blue points and the blue median values were obtained for events with modulation periods shorter than $20 \mathrm{~s}$. QP = quasiperiodic.

plots, that is, for long QP modulation periods. On the other hand, no such trend is apparent for short QP modulation periods in Figure 7a (AE index dependence) and only a weak trend is apparent in Figure $7 \mathrm{~b}$ (Dst index dependence). This suggests that QP events with short and long modulation periods may behave differently, and it might be worthy to investigate them separately. As it is clearly not possible to determine an exact threshold value separating "short" and "long" modulation periods, we use a modulation period of $20 \mathrm{~s}$ as a rough reasonable estimate of this value hereinafter. We note, however, that the results obtained are principally independent of this exact choice, and they would change only marginally if another threshold value in the interval between 15 and $30 \mathrm{~s}$ was used instead (not shown). Out of 2,172 events analyzed in this paper, modulation periods of 1,247 events were above $20 \mathrm{~s}$, whereas modulation periods of 925 events were shorter than $20 \mathrm{~s}$. The shortest observed QP modulation period was about $8 \mathrm{~s}$. The $20 \mathrm{~s}$ threshold is plotted by the horizontal red lines in Figures $7 \mathrm{a}$ and $7 \mathrm{~b}$. The horizontal black lines correspond to median modulation period values calculated separately for QP events with shorter and longer modulation periods over corresponding multiplicative factor of 2 in AE values and over intervals of $10 \mathrm{nT}$ in Dst values. It can be seen in Figure 7a that while the medians of short modulation periods are indeed nearly independent of the AE index, the medians of long modulation periods decrease with increasing AE index. Although the difference between the behavior of short and long modulation period events in Figure $7 \mathrm{~b}$ is not so pronounced, the modulation period decrease with increasing geomagnetic activity is still noticeably stronger for long modulation period events. The corresponding rank correlations for cases with long modulation periods are about -0.35 and 0.40 for $\mathrm{AE}$ and Dst indices, respectively.

The intensity of individual QP events as a function of AE and Dst indices is investigated in Figures 7c and $7 \mathrm{~d}$. The dependences obtained for short/long modulation period events are plotted by the blue/red col- 
(a)

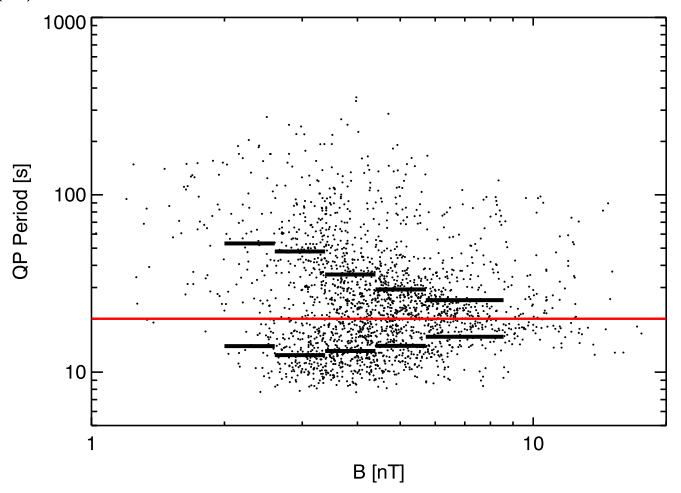

(c)

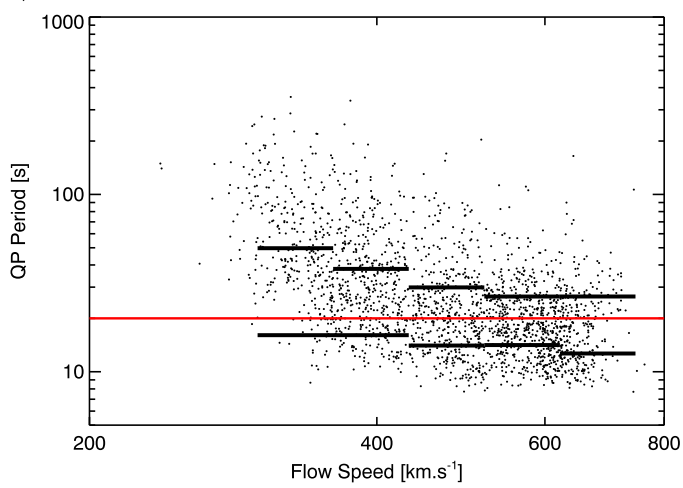

(b)

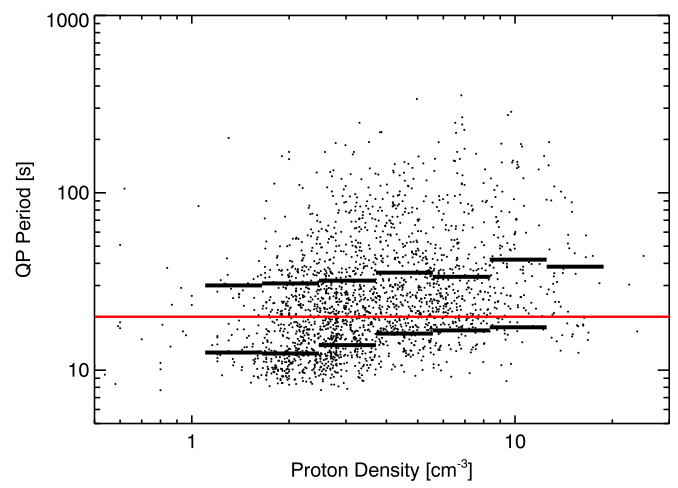

(d)

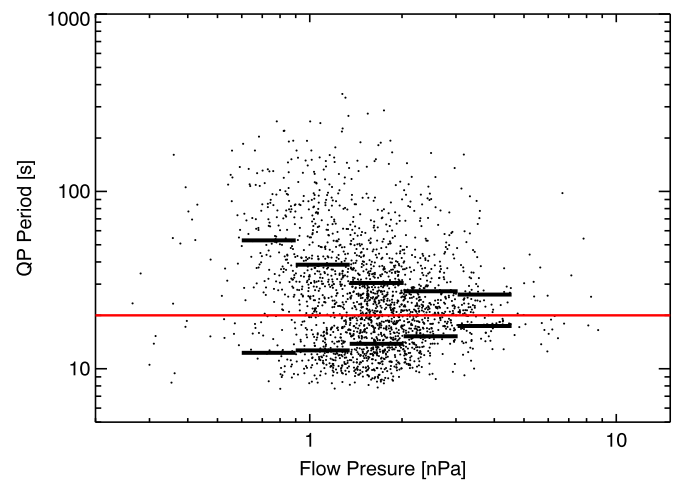

Figure 8. The same as Figure 6, but for the modulation period of QP events. The red horizontal lines mark the threshold modulation period value of $20 \mathrm{~s}$. The median values of QP modulation period shown by the black horizontal lines were calculated separately for events with shorter and longer modulation periods. QP = quasiperiodic.

ors, respectively. Similarly as for the modulation period dependences, there is principally no global trend observed for short modulation period events. On the other hand, although the scatter of individual data points is again very large, the median intensity of long modulation period events clearly increases with geomagnetic activity, by roughly an order of magnitude when the median values for events occurring during the lowest and the largest geomagnetic activity are compared. Note that-albeit their intensity is principally independent of the geomagnetic activity - the events with shorter modulation periods are generally more intense than the events with longer modulation periods. This is consistent with the results obtained by Hayosh et al. (2014), who showed that intensity of QP events decreases with increasing modulation periods. Additionally, the distribution of intensities of short modulation period events is significantly narrower than that obtained for long modulation period events. This might well be a consequence of the narrower range of periods considered, but it also possibly suggests that the events with short modulation periods are in some sense quite similar to each other, exhibiting a similar behavior and properties.

Figure 8 shows the dependences obtained for QP modulation periods as a function of solar wind parameters. The analysis was again done separately for events with modulation periods shorter/longer than $20 \mathrm{~s}$. The dots show results obtained for individual events, which are again largely scattered. The horizontal black lines correspond to median values calculated over the same intervals of solar wind parameters as in Figure 6. Figures $8 \mathrm{~b}$ and $8 \mathrm{c}$ show that the median modulation periods of QP events tend to increase with the solar wind proton number density and decrease with the solar wind flow speed. These global trends are observed both for short and long modulation period events. On the other hand, the dependences obtained for the IMF magnitude and for the solar wind flow pressure are rather different for short and long modulation period events. Specifically, Figures $8 \mathrm{a}$ and $8 \mathrm{~d}$ show that while the median modulation period of short modulation period events increases with the IMF magnitude and solar wind flow pressure, an opposite trend is observed for the median modulation period of long modulation period events. 
(a)

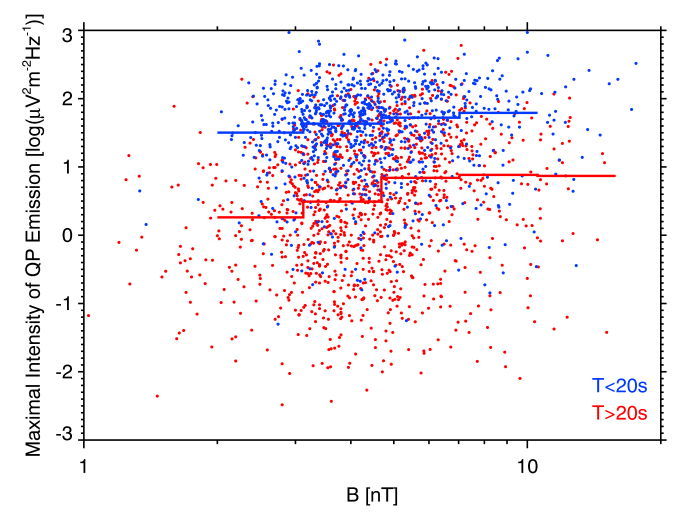

(c)

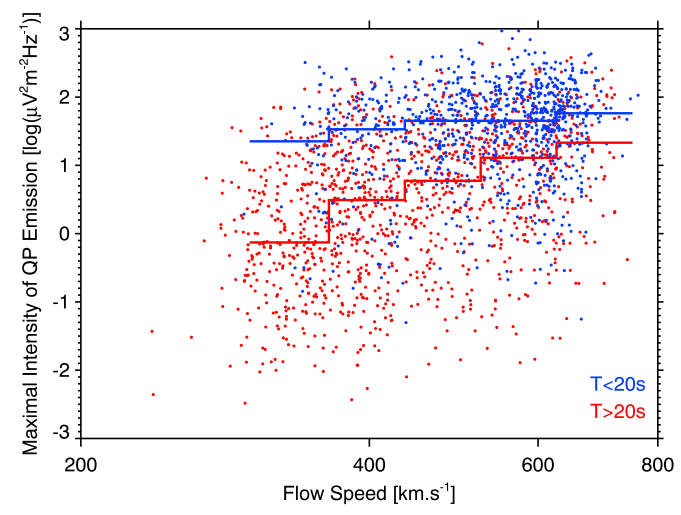

(b)

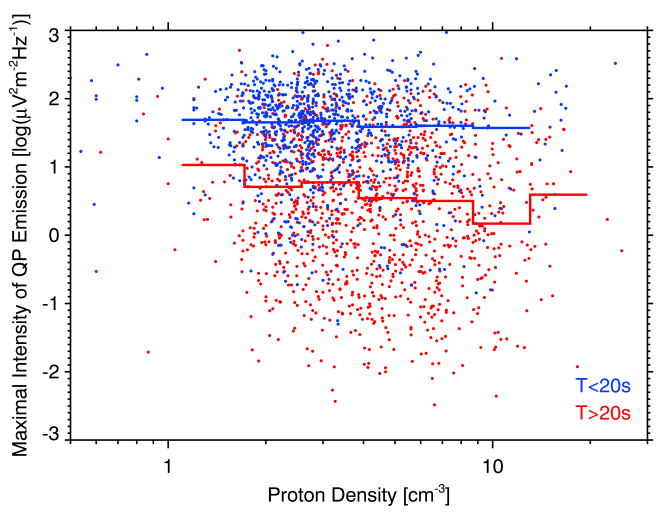

(d)

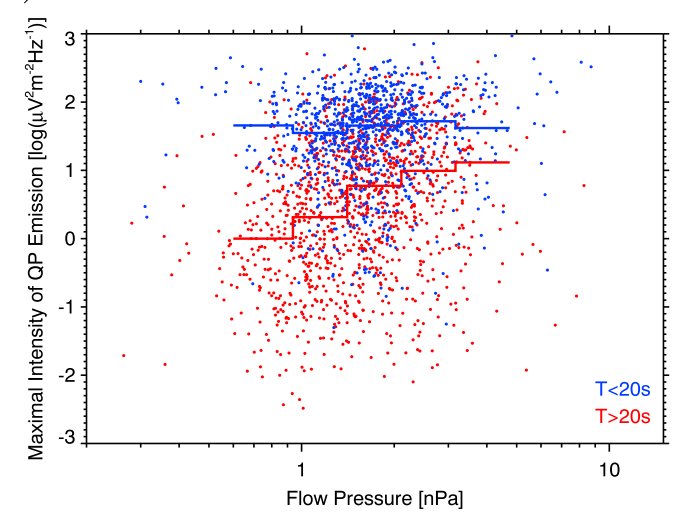

Figure 9. The same as Figure 6, but for the maximal intensity of QP events. The red points and the red median values were obtained for QP events with modulation periods longer than $20 \mathrm{~s}$. The blue points and the blue median values were obtained for QP events with modulation periods shorter than $20 \mathrm{~s}$. QP = quasiperiodic.

Dependences of QP intensities on solar wind parameters are investigated in Figure 9. The blue and red data points and medians were obtained for the events with short and long modulation periods, respectively. It can be seen that the trends obtained for events with short modulation periods are generally rather weak. The median intensity of this type of events tends to somewhat increase with the IMF magnitude and solar wind flow speed (rank correlation coefficients of 0.11 and 0.15 , respectively), while it possibly decreases with the solar wind proton number density (rank correlation coefficient of about -0.07). The median intensities of events with modulation periods longer than $20 \mathrm{~s}$ increase significantly with the solar wind flow speed and flow pressure (rank correlation coefficients of about 0.39 and 0.27 , respectively), while the individual results are again largely scattered. The relations of the median intensities of events with longer modulation periods to the IMF magnitude and proton number densities are quite weak, however, there appears to be a slight trend of the median intensity to decrease with increasing proton number density.

\subsection{MLR-QP Interrelationships and Dependence on the Whistler Occurrence Rate}

It is interesting to compare the properties of MLR and QP events observed at the same/similar times and to check for their possible relations. For this purpose, we consider the event properties to be potentially related if the events were observed within one DEMETER orbit from each other, that is, within less than about $2 \mathrm{hr}$, on the same dayside. Altogether, this condition resulted in 260 MLR-QP event pairs, whose properties are compared in Figure 10. Note that these event pairs appear to occur quite randomly; we were not able to identify any specific geomagnetic or other conditions which would favor their occurrence.

Figure 10a shows the dependence of the QP modulation period on the frequency spacing of corresponding MLR events. The black horizontal lines correspond to median values, and the analysis is again performed separately for QP events with short and long modulation periods. There seems to be basically no relation between the two parameters. Similarly, Figure 10b shows there is no relation of QP emission intensities and the frequency spacings of corresponding MLR events. 
(a)

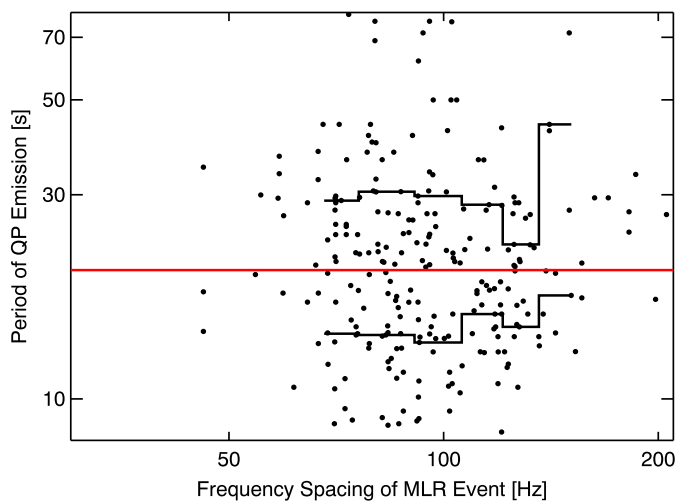

(b)

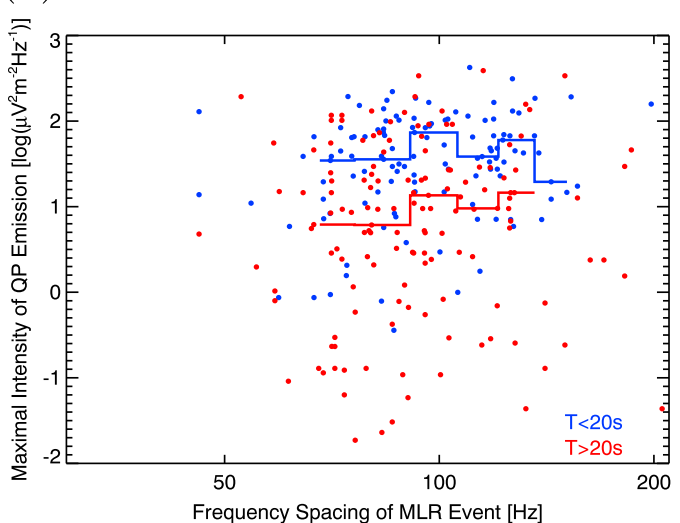

Figure 10. (a) Modulation periods of QP events as a function of frequency spacings of MLR events observed at the same times. The red line marks a threshold modulation period of $20 \mathrm{~s}$. The black horizontal lines show the median modulation period values calculated separately for events with shorter and longer modulation periods. (b) Maximal intensities of QP events as a function of the frequency spacing of MLR events observed at the same times. The red points and the red median values were obtained for QP events with modulation periods longer than $20 \mathrm{~s}$. The blue points and the blue median values were obtained for QP events with modulation periods shorter than $20 \mathrm{~s}$. $\mathrm{MLR}=$ magnetospheric line radiation; $\mathrm{QP}=$ quasiperiodic.

Finally, we investigated a possible influence of lightning generated whistlers detected by the neural network on board DEMETER on the event properties. Figure 11 uses the same format as Figures 5 and 7 to plot the event properties as a function of the average whistler occurrence rate calculated during the parts of half-orbits where the events were observed. It can be seen that the event parameters are quite independent of the whistler occurrence rate at geomagnetic latitudes between $40^{\circ}$ and $60^{\circ}$.

We further attempted to perform the same analysis separately for whistlers with low/middle/large dispersions. However, the event properties remained principally independent of the whistler occurrence rate irrespective of the whistler selection.

\section{Discussions}

The frequency spacing of MLR events was found to increase with the geomagnetic activity. This is consistent with the results obtained by Němec et al. (2012), which showed a relation between the frequency spacing and the model plasmapause location which itself depends on the geomagnetic activity. This might suggest that the radial distance of the source region is related to the plasmapause radial distance. During geomagnetically disturbed periods, when the plasmapause is located at lower radial distances, both the frequency spacing and drift of the events increase, possibly corresponding to shorter characteristic time scales at these lower radial distances. We note, however, that it is difficult to directly relate the observed frequency spacing (which is typically about $100 \mathrm{~Hz}$ ) to any of the characteristic plasma frequencies (Němec et al., 2012).

The dependences obtained for QP emissions are clearly different for QP events with short and long modulation periods, suggesting their different generation mechanism. Note that even the shortest QP modulation periods (about $8 \mathrm{~s}$ ) are too long to be due to periodic wave bouncing between the hemispheres, which typically corresponds to between 3 and 6 s (Manninen et al., 2014; Němec et al., 2009). The observed decrease of the modulation periods of longer period events with the geomagnetic activity is consistent with the ground-based case study by Manninen et al. (2013). They showed that the modulation periods of long-lasting QP events tend to decrease during substorm injections. This is in line with the predictions of the flow cyclotron maser theory (Demekhov \& Trakhtengerts, 1994; Pasmanik et al., 2004) for the times of enhanced energetic particle fluxes. Wave intensities at these times are also expected to be higher. Alternatively, assuming the generation mechanism based on ULF magnetic field pulsations, one might argue that at geomagnetically disturbed times the source region possibly moves to lower radial distances, the frequencies of ULF magnetic field pulsations increase accordingly, and QP emissions with shorter modulation periods are thus generated. We note, however, that the period of ULF magnetic field pulsations is generally 
(a)

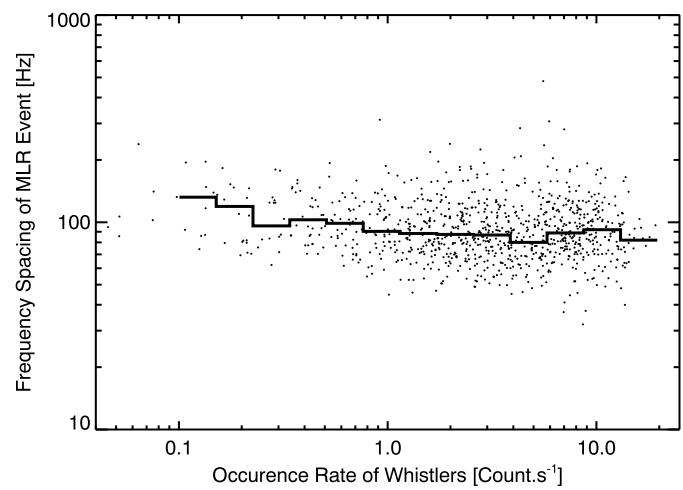

(b)

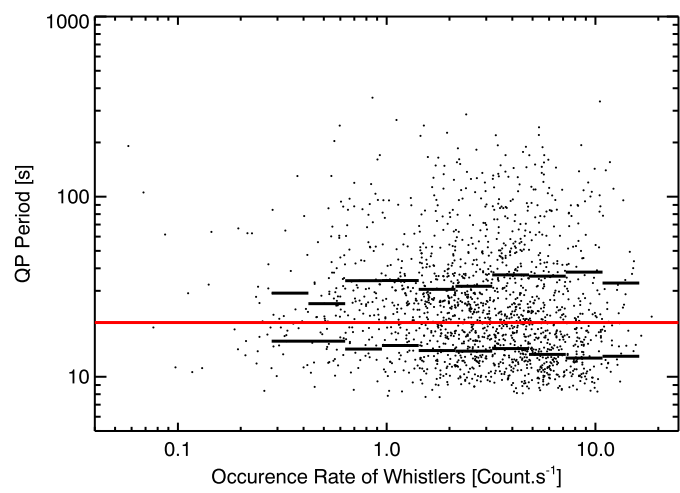

(c)

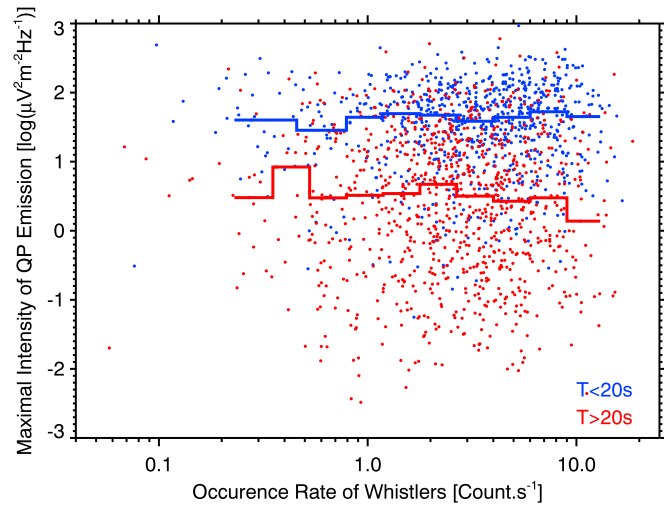

Figure 11. (a) MLR frequency spacing as a function of the average whistler occurrence rate. Each point corresponds to a single MLR event. Horizontal black lines correspond to median values in given whistler occurrence rate intervals. (b) QP modulation period as a function of the average whistler occurrence rate. Each point corresponds to a single event. The red horizontal line marks the modulation period of $20 \mathrm{~s}$. The horizontal black lines show median modulation period values calculated separately for short and long modulation period events. (c) Maximal intensity of QP events as a function of the average whistler occurrence rate. The red points and the red median values were obtained for events with modulation periods longer than $20 \mathrm{~s}$. The blue points and the blue median values were obtained for events with modulation periods shorter than $20 \mathrm{~s}$. The average whistler occurrence rate was computed at geomagnetic latitudes between $40^{\circ}$ and $60^{\circ}$ during the parts of half-orbits where the events were observed. MLR $=$ magnetospheric line radiation; $\mathrm{QP}=$ quasiperiodic. 
too large (e.g., Orr \& Matthew, 1971) to explain the events with short modulation periods, at least as far as only low-order field line oscillation harmonics are considered.

As for the dependence of the event properties on solar wind parameters, it is important to realize that due to their ultimate control of the geomagnetic activity, the obtained dependences cannot be completely separated from the analysis based on geomagnetic indices. Nevertheless, magnetospheric ULF pulsations are to some extent directly related to properties of the solar wind (Kepko et al., 2002; Mthembu et al., 2009). The frequencies of magnetospheric ULF pulsations are expected to be higher at the times of larger IMF magnitudes (Alford et al., 1996), which might be possibly consistent with the observed decrease of modulation periods of long modulation period QP events at the times of strong IMF. Their decrease with the increasing solar wind flow speed/pressure might be then understood in terms of the compressed magnetosphere resulting in higher frequencies of eigenoscillations of the magnetopause-plasmasphere resonator (e.g., Claudepierre et al., 2009). The increase of QP emission intensities with the solar wind flow speed is understandable in terms of the aforementioned geomagnetic activity dependence, taking into account that the solar wind-magnetosphere energy transport is generally more effective at the times of larger solar wind flow speeds (e.g., Finch \& Lockwood, 2007). The dependences obtained for the solar wind flow pressure and proton number density then possibly simply stem from the interrelations between solar wind parameters.

All the obtained results strongly indicate that QP events with short and long modulation periods behave quite differently. While the dependences obtained for QP events with modulation periods shorter than about $20 \mathrm{~s}$ are very weak if any, the dependences obtained for QP events with modulation periods longer than about $20 \mathrm{~s}$ are generally much stronger. This suggests that short and long modulation period QP events might be generated in different ways.

There appears to be principally no relation between the properties of MLR and QP events observed at about the same times. Additionally, although the events are at times observed simultaneously or at least during the same half-orbits, this rather appears to be a coincidence than a systematic feature. Also, while DEMETER observes QP emissions nearly exclusively during the daytime (Hayosh et al., 2014), daytime observations of MLR are only by a factor of about 1.7 more frequent than nighttime MLR observations (Bezděková et al., 2015). All these results suggest that although a possible relation between MLR and QP events would be tempting, they are most likely not related in any identifiable way.

Ho (1973) suggested that QP emissions can be at times initiated by lightning generated whistlers, and, moreover, that lightning generated whistlers may disrupt well-behaved QP patterns by suddenly increasing the QP period. This might be possibly explained in terms of flow cyclotron maser theory (Demekhov \& Trakhtengerts, 1994; Pasmanik et al., 2004), assuming a significant lightning induced electron precipitation (Gemelos et al., 2009). However, our results show that a possible influence of the lightning generated whistlers on the QP event properties is very small, if any. This might be possibly explained by the fact that while the whistler occurrence rate is evaluated at geomagnetic latitudes between $40^{\circ}$ and $60^{\circ}$, the source of the QP emissions itself may be located at different magnetic field lines and spatially rather limited. Consequently, it is difficult to properly evaluate the number of whistlers passing through the source and possibly affecting the wave generation conditions there.

\section{Conclusions}

We presented a statistical study of properties of MLR and QP events observed during the whole mission of the low-altitude DEMETER spacecraft. The large numbers of identified MLR $(1,230)$ and QP $(2,264)$ events represent a unique data set optimally suited for such studies.

We showed that the frequency drift of MLR events with larger-frequency spacings is typically larger and that the frequency spacing of MLR events is larger during geomagnetically distributed periods and that it is also larger at the times of higher solar wind flow speeds.

Different behavior of QP emissions with short and long modulation periods (with a threshold modulation period $20 \mathrm{~s}$ ) was further demonstrated. While the global properties of QP emissions with long modulation periods are significantly influenced by geomagnetic activity and solar wind parameters (especially by the solar wind flow speed and the IMF magnitude), events with shorter modulation periods are rather indepen- 
Acknowledgments

The authors would like to thank all the engineers from CNES and scientific laboratories (CBK, IRAP, LPC2E, LPP, and SSD of ESTEC) who assisted in the DEMETER mission and helped to its excellent results. The whole DEMETER data set is available from https://sipad-cdpp.cnes. frllignorespaceswebsite. OMNI solar wind data are available from https:// omniweb.gsfc.nasa.gov/ website; $\mathrm{AE}$ and Dst indices

values are available from http://wdc kugi.kyoto-u.ac.jp/

dstae/index.html website. The authors were supported by the Czech Science Foundation (GAČR) under Contracts 18-00844S and 17-07027S. The work of O. S. was further supported by the Praemium Academiae award from the Czech Academy of Sciences. dent of them. This suggests that QP events with short and long modulation periods might be generated by different mechanisms.

There is principally no relation between the properties of MLR and QP events observed at the same times. The properties of neither MLR nor QP events seem to be affected by the whistler occurrence rates at the times of the events.

\section{References}

Alford, J., Engebretson, M., Arnoldy, R., \& Inan, U. (1996). Frequency variations of quasi-periodic ELF-VLF emissions: A possible new ground-based diagnostic of the outer high-latitude magnetosphere. Journal of Geophysical Research, 101(A1), 83-97. https://doi.org/10. 1029/95JA02223

Bell, T. F., Luette, J. P., \& Inan, U. S. (1982). ISEE 1 observations of VLF line radiation in the Earth's magnetosphere. Journal of Geophysical Research, 87(A5), 3530-3536. https://doi.org/10.1029/JA087iA05p03530

Berthelier, J. J., Godefroy, M., Leblanc, F., Malingre, M., Menvielle, M., Lagoutte, D., et al. (2006). ICE. The electric field experiment on DEMETER. Planetary and Space Science, 54, 456-471. https://doi.org/10.1016/j.pss.2005.10.016

Bezděková, B., Němec, F., Parrot, M., Santolík, O., \& Kruparova, O. (2015). Magnetospheric line radiation: 6.5 years of observations by the DEMETER spacecraft. Journal of Geophysical Research: Space Physics, 120, 9442-9456. https://doi.org/10.1002/2015JA021246

Bullough, K. (1995). Handbook of atmospheric electrodynamics, (Vol. 2, pp. 291-332). Boca Raton, FL: CRC Press.

Carson, W. B., Koch, J. A., Pope, J. H., \& Gallet, R. M. (1965). Long period very low frequency emission pulsations. Journal of Geophysical Research, 70(17), 4293-4303. https://doi.org/10.1029/JZ070i017p04293

Chen, L. (1974). Theory of ULF modulation of VLF emissions. Geophysical Research Letters, 1(2), 73-75. https://doi.org/10.1029/ GL001i002p00073

Claudepierre, S. G., Wiltberger, M., Elkington, S. R., Lotko, W., \& Hudson, M. K. (2009). Magnetospheric cavity modes driven by solar wind dynamic pressure fluctuations. Geophysical Research Letters, 36, L13101. https://doi.org/10.1029/2009GL039045

Demekhov, A. G., \& Trakhtengerts, V. Y. (1994). A mechanism of formation of pulsating aurorae. Journal of Geophysical Research, 99(4), 5831-5841. https://doi.org/10.1029/93JA01804

Elie, F., Hayakawa, M., Parrot, M., Pinon, J. L., \& Lefeuvre, F. (1999). Neural network system for the analysis of transient phenomena on board the DEMETER micro-satellite. IEICE TRANSACTIONS on Fundamentals of Electronics, Communications and Computer Sciences, 82(8), 1575-1581.

Engebretson, M. J., Posch, J. L., Halford, A. J., Shelburne, G. A., Smith, A. J., Spasojevic, M., et al. (2004). Latitudinal and seasonal variations of quasiperiodic and periodic VLF emissions in the outer magnetosphere. Journal of Geophysical Research, 109, A05216. https://doi.org/ 10.1029/2003JA010335

Finch, I., \& Lockwood, M. (2007). Solar wind-magnetosphere coupling functions on timescales of 1 day to 1 year. Annals of Geophysics, 25, 495-506. https://doi.org/10.5194/angeo-25-495-2007

Gemelos, E. S., Inan, U. S., Walt, M., Parrot, M., \& Sauvaud, J. A. (2009). Seasonal dependence of energetic electron precipitation: Evidence for a global role of lightning. Geophysical Research Letters, 36, L21107. https://doi.org/10.1029/2009GL040396

Gołkowski, M., \& Inan, U. S. (2008). Multistation observations of ELF/VLF whistler mode chorus. Journal of Geophysical Research, 113 , A08210. https://doi.org/10.1029/2007JA012977

Hayosh, M., Němec, F., Santolík, O., \& Parrot, M. (2014). Statistical investigation of VLF quasiperiodic emissions measured by the DEMETER spacecraft. Journal of Geophysical Research: Space Physics, 119, 8063-8072. https://doi.org/10.1002/2013JA019731

Hayosh, M., Němec, F., Santolík, O., \& Parrot, M. (2016). Propagation properties of quasi-periodic VLF emissions observed by the DEMETER spacecraft. Journal of Geophysical Research: Space Physics, 43, 1007-1014. https://doi.org/10.1002/2015GL067373

Helliwell, R. A., Katsufrakis, J. P., Bell, T. F., \& Raghuram, R. (1975). VLF line radiation in the Earth's magnetosphere and its association with power system radiation. Journal of Geophysical Research, 80, 4249-4258. https://doi.org/10.1029/JA080i031p04249

Ho, D. (1973). Interaction between whistlers and quasi-periodic VLF emissions. Journal of Geophysical Research, 78(31), 7347-7356. https:// doi.org/10.1029/JA078i031p07347

Kepko, L., Spence, H. E., \& Singer, H. J. (2002). ULF waves in the solar wind as direct drivers of magnetospheric pulsations. Geophysical Research Letters, 29(8), 1197. https://doi.org/10.1029/2001GL014405

Kimura, I. (1974). Interrelation between VLF and ULF emissions. Space Science Reviews, 16, 389-411. https://doi.org/10.1007/BF00171565

Kitamura, T., Jacobs, J. A., Watanabe, T., \& Flint, J. R. B. (1969). An investigation of quasi-periodic VLF emissions. Journal of Geophysical Research, 74(24), 5652-5664. https://doi.org/10.1029/JA074i024p05652

Manninen, J. (2005). Some aspects of ELF-VLF emissions in geophysical research, pp. 53-84, 85-110. Sodankylä, Finland: Sodankylä Geophysical Observatory Publications. http://www.sgo.fi/Publications/SGO/thesis/ManninenJyrki.pdf.

Manninen, J., Demekhov, A. G., Titova, E. E., Kozlovsky, A. E., \& Pasmanik, D. L. (2014). Quasiperiodic VLF emissions with short-period modulation and their relationship to whistlers: A case study. Journal of Geophysical Research: Space Physics, 119, 3544-3557. https://doi. org/10.1002/2013JA019743

Manninen, J., Kleimenova, N. G., \& Kozyreva, O. V. (2012). New type of ensemble of quasi-periodic, long-lasting VLF emissions at the auroral zone. Annales Geophysicae, 30(12), 1655. https://doi.org/10.5194/angeo-30-1655-2012

Manninen, J., Kleimenova, N. G., Kozyreva, O. V., Bespalov, P. A., \& Kozlovsky, A. E. (2013). Non-typical ground-based quasi-periodic VLF emissions observed at $L \sim 5.3$ under quiet geomagnetic conditions at night. Journal of Atmospheric and Solar-Terrestrial Physics, 99, 123-128. https://doi.org/10.1016/j.jastp.2012.05.007

Manninen, J., Kleimenova, N., Turunen, T., \& Gromova, L. (2018). New high-frequency (7-12 kHz) quasi-periodic VLF emissions observed on the ground at $L \sim 5.5$. Annales Geophysicae, 36(3), 915-923. https://doi.org/10.5194/angeo-36-915-2018

Manninen, J., Titova, E. E., Demekhov, A. G., Kozlovsky, A. E., \& Pasmanik, D. L. (2014). Quasiperiodic VLF emissions: Analysis of periods on different timescales. Cosmic Research, 52(1), 61-67. https://doi.org/10.1134/S0010952514010055 
Martinez-Calderon, C., Shiokawa, K., Miyoshi, Y., Keika, K., Ozaki, M., Schofield, I., \& Kurth, W. S. (2016). ELF/VLF wave propagation at subauroral latitudes: Conjugate observation between the ground and Van Allen Probes A. Journal of Geophysical Research: Space Physics, 121, 5384-5393. https://doi.org/10.1002/2015JA022264

Martinez-Calderon, C., Shiokawa, K., Miyoshi, Y., Ozaki, M., Schofield, I., \& Connors, M. (2015). Statistical study of ELF/VLF emissions at subauroral latitudes in Athabasca, Canada. Journal of Geophysical Research: Space Physics, 120, 8455-8469. https://doi.org/10.1002/ 2015JA021347

Morrison, K. (1990). Quasi-periodic VLF emissions and concurrent magnetic pulsations seen at $L=4$. Planetary and Space Science, 38(12), 1555-1565. https://doi.org/10.1016/0032-0633(90)90161-I

Mthembu, S. H., Malinga, S. B., Walker, A. D. M., \& Magnus, L. (2009). Characterization of ultra low frequency ULF pulsations and the investigation of their possible source. Annales Geophysicae, 27, 3287-3296. https://doi.org/10.5194/angeo-27-3287-2009

Němec, F., Bezděková, B., Manninen, J., Parrot, M., Santolík, O., Hayosh, M., \& Turunen, T. (2016). Conjugate observations of a remarkable quasiperiodic event by the low-altitude DEMETER spacecraft and ground-based instruments. Journal of Geophysical Research: Space Physics, 121, 8790-8803. https://doi.org/10.1002/2016JA022968

Němec, F., Hospodarsky, G. B., Bezděková, B., Demekhov, A. G., Pasmanik, D. L., Santolík, O., et al. (2018). Quasiperiodic whistler mode emissions observed by the Van Allen Probes spacecraft. Journal of Geophysical Research: Space Physics, 123, 8969-8982. https://doi.org/ 10.1029/2018JA026058

Němec, F., Parrot, M., \& Santolík, O. (2012). Detailed properties of magnetospheric line radiation events observed by the DEMETER spacecraft. Journal of Geophysical Research, 117, A05210. https://doi.org/10.1029/2012JA017517

Němec, F., Parrot, M., Santolík, O., Rodger, C. J., Rycroft, M. J., Hayosh, M., et al. (2009). Survey of magnetospheric line radiation events observed by the DEMETER spacecraft. Journal of Geophysical Research, 114, A05203. https://doi.org/10.1029/2008JA014016

Němec, F., Raita, T., Parrot, M., Santolík, O., \& Turunen, T. (2009). Conjugate observations on board a satellite and on the ground of a remarkable MLR-like event. Geophysical Research Letters, 36, L22103. https://doi.org/10.1029/2009GL040974

Němec, F., Santolík, O., Boardsen, S. A., Hospodarsky, G. B., \& Kurth, W. S. (2018). Equatorial noise with quasiperiodic modulation: Multipoint observations by the Van Allen Probes spacecraft. Journal of Geophysical Research: Space Physics, 123, 4809-4819. https://doi. org/10.1029/2018JA025482

Němec, F., Santolík, O., Hayosh, M., Darrouzet, F., \& Cornilleau-Wehrlin, N. (2018). Detailed properties of equatorial noise with quasiperiodic modulation. Journal of Geophysical Research: Space Physics, 123, 5344-5355. https://doi.org/10.1029/2018JA025382

Němec, F., Santolík, O., Hrbáčková, Z., Pickett, J. S., \& Cornilleau-Wehrlin, N. (2015). Equatorial noise emissions with quasiperiodic modulation of wave intensity. Journal of Geophysical Research: Space Physics, 120, 2649-2661. https://doi.org/10.1002/2014JA020816

Němec, F., Santolík, O., Parrot, M., \& Berthelier, J. J. (2007). Comparison of magnetospheric line radiation and power line harmonic radiation: A systematic survey using the DEMETER spacecraft. Journal of Geophysical Research, 112, A04301. https://doi.org/10.1029/ 2006JA012134

Němec, F., Santolík, O., Parrot, M., \& Pickett, J. S. (2012). Magnetospheric line radiation event observed simultaneously on board Cluster 1, Cluster 2 and DEMETER Spacecraft. Geophysical Research Letters, 39, L18103. https://doi.org/10.1029/2012GL053132

Němec, F., Santolík, O., Parrot, M., Pickett, J. S., Hayosh, M., \& Cornilleau-Wehrlin, N. (2013). Conjugate observations of quasi-periodic emissions by Cluster and DEMETER spacecraft. Journal of Geophysical Research: Space Physics, 118, 198-208. https://doi.org/10.1029/ 2012JA018380

Němec, F., Santolík, O., Pickett, J. S., Parrot, M., \& Cornilleau-Wehrlin, N. (2013). Quasiperiodic emissions observed by the Cluster spacecraft and their association with ULF magnetic pulsations. Journal of Geophysical Research: Space Physics, 118, 4210-4220. https:// doi.org/10.1002/jgra.50406

Orr, D., \& Matthew, J. A. D. (1971). The variation of geomagnetic micropulsation periods with latitude and the plasmapause. Planetary and Space Science, 19, 897-905. https://doi.org/10.1016/0032-0633(71)90141-3

Parrot, M., Manninen, J., Santolik, O., Nemec, F., Turunen, T., Raita, T., \& Macusova, E. (2007). Simultaneous observation on board a satellite and on the ground of large-scale magnetospheric line radiation. Geophysical Research Letters, 34, L19102. https://doi.org/10. 1029/2007GL030630

Parrot, M., Němec, F., Santolík, O., \& Berthelier, J. J. (2005). ELF magnetospheric lines observed by DEMETER. Annales Geophysicae, 23, 3301-3311. https://doi.org/10.5194/angeo-23-3301-2005

Parrot, M., Němec, F., Santolík, O., \& Cornilleau-Wehrlin, N. (2016). Equatorial noise emissions with a quasiperiodic modulation observed by DEMETER at harmonics of the O+ ion gyrofrequency. Journal of Geophysical Research: Space Physics, 121, 10-289. https://doi.org/ 10.1002/2016JA022989

Pasmanik, D. L., Demekhov, A. G., Trakhtengerts, V. Y., \& Parrot, M. (2004). Modeling whistler wave generation regimes in magnetospheric cyclotron maser. Annales Geophysicae, 22, 3561-3570. https://doi.org/10.5194/angeo-22-3561-2004

Pasmanik, D. L., Titova, E. E., Demekhov, A. G., Trakhtengerts, V. Y., Santolík, O., Jiříček, F., et al. (2004). Quasiperiodic ELF/VLF wave emissions in the Earth's magnetosphere: Comparison of satellite observations and modelling. Annales Geophysicae, 22, 4351-4361. https://doi.org/10.5194/angeo-22-4351-2004

Rodger, C. J., Clilverd, M. A., Yearby, K. H., \& Smith, A. J. (1999). Magnetospheric line radiation observations at Halley, Antarctica. Journal of Geophysical Research, 104(A8), 17,441-17,447. https://doi.org/10.1029/1999JA900153

Rodger, C. J., Clilverd, M. A., Yearby, K., \& Smith, A. J. (2000a). Is magnetospheric line radiation man-made? Journal of Geophysical Research, 105(A7), 15,981-15,990. https://doi.org/10.1029/1999JA000413

Rodger, C. J., Clilverd, M. A., Yearby, K. H., \& Smith, A. J. (2000b). Temporal properties of magnetospheric line radiation. Journal of Geophysical Research, 105(A1), 329-336. https://doi.org/10.1029/1999JA900420

Rodger, C. J., Thomson, N. R., \& Dowden, R. L. (1995). VLF line radiation observed by satellite. Journal of Geophysical Research, 100(A4), 5681-5689. https://doi.org/10.1029/94JA02865

Sato, N., \& Fukunishi, H. (1981). Interaction between ELF-VLF emissions and magnetic pulsations: Classification of quasiperiodic ELF-VLF emissions based on frequency-time spectra. Journal of Geophysical Research, 86(A1), 19-29. https://doi.org/10.1029/ JA086iA01p00019

Sato, N., Hayashi, K., Kokubun, S., Oguti, T., \& Fukunishi, H. (1974). Relationships between quasiperiodic VLF emission and geomagnetic pulsation. Journal of Atmospheric and Solar-Terrestrial Physics, 36, 1515-1526. https://doi.org/10.1016/0021-9169(74)90229-3

Sazhin, S. S., \& Hayakawa, M. (1994). Periodic and quasiperiodic VLF emissions. Journal of Atmospheric and Terrestrial Physics, 56, 735-753. https://doi.org/10.1016/0021-9169(94)90130-9 
Titova, E. E., Kozelov, B. V., Demekhov, A. G., Manninen, J., Santolík, O., Kletzing, C. A., \& Reeves, G. (2015). Identification of the source of quasiperiodic VLF emissions using ground-based and Van Allen Probes satellite observations. Geophysical Research Letters, 42, 6137-6145. https://doi.org/10.1002/2015GL064911

Tixier, M., \& Cornilleau-Wehrlin, N. (1986). How are the VLF quasiperiodic emissions controlled by harmonics of field line oscillations? The results of a comparison between ground and GEOS satellites measurements. Journal of Geophysical Research, 91(A6), 6899-6919. https://doi.org/10.1029/JA091iA06p06899

Trakhtengerts, V. Y., Tagirov, V. R., \& Chernous, S. A. (1986). A flowing cyclotron maser and impulsive VLF emissions. Geomagnetism and Aeronomy, 26, 99-106. 